\title{
Synthesis, Characterization, Molecular Docking Studies and Antimicrobial Evaluation of N-Benzimidazol-1-YI-Methyl-Benzamide Derivatives
}

\author{
Ritchu Sethi' ${ }^{1}$, Sandeep Arora', Deepika Saini ${ }^{2}$ and Sandeep Jain ${ }^{2^{*}}$ \\ ${ }^{1}$ Chitkara College of Pharmacy, Chitkara University, Rajpura, Distt. Patiala-140401, INDIA. \\ ${ }^{2}$ Department of Pharmaceutical Sciences Guru Jambheshwar University, Hissar-125001, INDIA.
}

\begin{abstract}
$\mathrm{N}$-benzimidazol-1-yl-methyl-benzamide derivatives (3a-3x) were synthesized by Mannich reaction and evaluated for in vitro antimicrobial activity against Escherichia coli, Pseudomonas aeruginosa, Bacillus subtilis, Staphylococcus aureus, Candida albicans and Aspergillus niger. The structures of novel target compounds were elucidated by spectral and analytical techniques. Among the synthesized derivatives, 3o N-(2-(2-chloro-phenyl)benzimidazol-1-y/methyl]-benzamide, 3q N-[2-(4-chloro-phenyl)-benzimidazol-1ylmethyl]benzamide and $3 \mathrm{r} N$-[2-(2-bromo-phenyl)-benzimidazol-1-ylmethyl]-benzamide were found to be most effective antimicrobial compounds. Clotrimazole and ciprofloxacin were used as reference antimicrobial agents. Further, in silico studies were carried out to define the interaction of the title compounds with microbial protein.
\end{abstract}

Key words: Mannich bases, Benzimidazole, Antibacterial activity, Antifungal activity, Docking study.

\section{INTRODUCTION}

Antimicrobial resistance is an evolving predicament in treating the patients, and causes several deaths every year. ${ }^{1}$ The main cause of microbial resistance is the mutations or transfer of resistant genes between organisms. ${ }^{2}$ Resistant microbes may require other medications or elevated doses associated with more side effects, some of which may be life alarming. The chronic use of antibiotics is escalating the rates of infections, due to antibiotic resistance. As resistance to antibiotics becomes more common, the development of novel, effective and inimitable antimicrobial agents is the only superlative way to conquer microbial resistance and develop effective therapies.

Despite of several attempts to build up new structural analogues in the seek for more effective antimicrobials, the benzimidazoles still remain the most flexible class of com- pounds against microbes. ${ }^{3-5} 2$ - substituted benzimidazole and its derivatives have attracted great attention for the past few decades due to their chemotherapeutic values and therefore, are useful compounds for further molecular discovery. These derivatives show biological activities such as antimicrobial, ${ }^{6}$ anti-allergic, ${ }^{7}$ PARP (poly ADP ribose polymerase) inhibitors- as anticancer agents, ${ }^{8}$ as cytomegalovirus (HCMV) inhibitors, ${ }^{9}$ antiulcer, ${ }^{10}$ anti inflammatory, ${ }^{11}$ and as antihistaminics. ${ }^{12}$

On the other side mannich bases are the end products of mannich reaction and are known as beta amino ketone carrying compounds..$^{13}$ Mannich bases are very reactive because of the introduction of basic functional group which renders the derivative in aqueous solvent and can be changed into several other compounds. ${ }^{14}$ Literature
Submission Date : 14-03-2016 Revision Date : 12-05-2016 Accepted Date : :02-06-2016

DOI: 10.5530/ijper.50.3.16

Correspondence:

Sandeep Jain,

Department of Pharma-

ceutical Sciences, Guru Jambheshwar University of

Science and Technology,

Hissar, INDIA.

Mobile: +919416498857

E-mail: drsjain1969@yahoo.

co.in

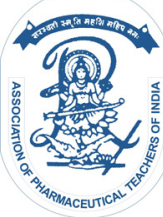

www.ijper.org 
revealed that mannich bases have been reported to possess analgesic, ${ }^{15}$ anti-inflammatory, ${ }^{16,17}$ anticancer, ${ }^{18,19}$ anticonvulsant, ${ }^{20}$ antibacterial, ${ }^{21,22}$ antifungal, ${ }^{22,23}$ and several other activities.

Keeping the above facts in mind and as part of continuation of our research on Mannich bases, ${ }^{24}$ we hereby report the synthesis and antimicrobial evaluation of mannich bases of 2-substituted benzimidazole derivatives. Their structural configuration was elucidated with the elemental and spectral analysis. Synthesized novel compounds derived from mannich reaction were further evaluated for antimicrobial activity and then screened by in silico method to study drug receptor interaction between novel compounds and microbial protein.

\section{MATERIALS AND METHODS}

Chemicals were procured from Sigma Aldrich, USA. Melting points of the synthesized compounds were determined by open capillary tubes and were uncorrected. The purity of the synthesized compounds was determined by thin layer chromatography on precoated silica gel $G$ plates with visualization by iodine vapours/ UV chamber. Infrared spectra ( $\mathrm{KBr}$ pellets) were recorded on Perkin Elmer Spectrum FTIR spectrophotometer. Proton NMR spectra were recorded on Bruker Avance II 400 NMR Spectrometer using DMSO-d6 as a solvent and TMS (tetra methyl silane) as internal standard (chemical shift in $\delta \mathrm{ppm}$ ). Elemental analyses were carried out using Eager Xperience CHN analyzer at Panjab University, Chandigarh. Mass spectra were taken on Q-TOF MICROMASS Spectrometer (WATERS, USA).

\section{Chemistry}

Mannich Bases of 2-substituted benzimidazole were synthesized by the reaction of 2-substituted benzimidazole (secondary amine), formalin and benzamide (active hydrogen compound) (3a-x). 2-substituted benzimidazoles (2a-x) were prepared by the reaction of ortho-phenylenediamine with substituted carboxylic acid and with substituted aromatic aldehyde respectively.

\section{General schemes for the synthesis of compounds}

\section{General method}

The title compounds were prepared by the following steps.

Synthesis of 2-substituted benzimidazoles from ortho-phenylenediamine dihydrochloride $[2(a-f), 2 b, 2 j, 2 v]$

2 -substituted benzimidazoles were synthesized by the reaction of o-phenylenediamine dihydrochloride with substituted carboxylic acid by the method described in literature. $^{25}$
Synthesis of 2-substituted benzimidazoles from ortho-phenylenediamine $[2(k-x), 2 i, 2 g]$

2- substituted benzimidazoles were synthesized by the reaction of o-phenylenediamine and substituted benzaldehyde by the procedure reported in literature. ${ }^{26-28}$

\section{Synthesis of mannich base of 2-substituted benzimidazoles with} benzamide $[3(a-x)]$

2- substituted benzimidazole ( 0.01 mole) was added to the ethanolic solution of benzamide $(0.01 \mathrm{~mole})$. Formaldehyde (37\%) ( 0.01 mole) was added and the reaction mixture was then adjusted to the $\mathrm{pH}$ of 3.5 with conc. $\mathrm{HCl}$. The mixture was kept at efficient ice cooling for half an hour. Then it was refluxed with stirring at $80^{\circ} \mathrm{C}$ for 10-12 hrs. Formaldehyde (37\%) was added to the reaction mixture in portions for completion of the reaction. End of reaction was monitored by TLC. Solvent system- $\mathrm{CHCl}_{3}: \mathrm{CH}_{3} \mathrm{OH}$; 9.5: 0.5. Reaction mixture was kept in refrigerator overnight. Solvent was evaporated under reduced pressure and product was collected, washed with water and recrystallized from ethanol.

Similar type of procedures and equimolar quantities of reactants were used and 24 novel compounds were synthesized. Synthetic route for synthesis of novel compounds is shown in the general scheme. Physical data of synthesized compounds is given in Table 1.

\section{Spectral data}

$\mathrm{N}$-(Benzimidazol-1-yl methyl)-benzamide. (3a)

White crystals, mp $198-199^{\circ} \mathrm{C}, \mathrm{IR}(\mathrm{KBr}, \mathrm{cm}-1) 3307$ $(\mathrm{N}-\mathrm{H}), 1486(\mathrm{C}=\mathrm{N}), 3053 \mathrm{C}-\mathrm{H}\left(\mathrm{CH}_{2}\right), 2965$ (C-H, Ar.) $1526(\mathrm{C}=\mathrm{C}), 1634(\mathrm{C}=\mathrm{O}), 675-870(\mathrm{CH}$ bend.Ar). $1 \mathrm{H}$ NMR (400 MHz, DMSO-d ${ }_{6}, \delta$ ppm) 7.39-7.91 (m, 10H, ArH), 4.98 (s, 2H, $\left.\mathrm{NCH}_{2} \mathrm{~N}\right), 8.70$ (s, 1H, NH). Anal Calcd for $\mathrm{C}_{15} \mathrm{H}_{13} \mathrm{~N}_{3} \mathrm{O}$ : C $71.70, \mathrm{H} 5.21, \mathrm{~N} 16.72$; found C 70.84, H 5.14, N 15.94.

\section{N-(2-methyl-benzimidazol-1-yl methyl)-benzamide (3b)}

White crystals, mp $180-182^{\circ} \mathrm{C}, \mathrm{IR}(\mathrm{KBr}, \mathrm{cm}-1) 3308$ $(\mathrm{N}-\mathrm{H}), 1487(\mathrm{C}=\mathrm{N}), 3055 \mathrm{C}-\mathrm{H}\left(\mathrm{CH}_{2}\right), 2966$ (C-H, Ar.), $1526(\mathrm{C}=\mathrm{C}), 1634(\mathrm{C}=\mathrm{O})$, 675-870 (CH bend.Ar). $1 \mathrm{H}$ NMR (400 MHz, DMSO-d,$\delta$ ppm) 7.3-7.9 (m, 9H, $\mathrm{ArH}), 4.89$ (s, $\left.2 \mathrm{H}, \mathrm{NCH}_{2} \mathrm{~N}\right), 8.8(\mathrm{~s}, 1 \mathrm{H}, \mathrm{NH}), 2.80$ (s, $3 \mathrm{H}, \mathrm{CH}_{3}$ ) Anal Calcd for $\mathrm{C}_{16} \mathrm{H}_{15} \mathrm{~N}_{3} \mathrm{O}: \mathrm{C} 72.43, \mathrm{H} 5.7$, $\mathrm{N}$ 15.84; found C 69.84, H 5.54, N 15.69. MS: $\mathrm{m} / \mathrm{z}=$ $266.2(\mathrm{M}+1)$.

\section{$\mathrm{N}$-(2-chloromethyl-benzimidazol-1-yl methyl)- benzamide (3c)}

Yellow crystals; mp. $190-191^{\circ} \mathrm{C}, \mathrm{IR}$ (KBr, cm-1) 3308 $(\mathrm{N}-\mathrm{H}), 1485(\mathrm{C}=\mathrm{N}), 3055 \mathrm{C}-\mathrm{H}\left(\mathrm{CH}_{2}\right), 2966(\mathrm{C}-\mathrm{H}, \mathrm{Ar}$. 1526 (C=C), 1634 (C=O), 789 (C-Cl), 675-870 (CH bend.Ar). 1HNMR (400 MHz, DMSO-d, $8 p p m)$, 


\begin{tabular}{|c|c|c|c|c|c|c|}
\hline Compound & $\mathbf{R}$ & Molecular formula & $\begin{array}{c}\text { Molecular } \\
\text { weight }\end{array}$ & $\begin{array}{c}\text { Melting } \\
\text { Point }\left({ }^{\circ} \mathrm{C}\right)\end{array}$ & $\begin{array}{c}\text { Yield } \\
(\%)\end{array}$ & $R_{f}$ value \\
\hline $3 a$ & $\mathrm{H}-$ & $\mathrm{C}_{15} \mathrm{H}_{13} \mathrm{~N}_{3} \mathrm{O}$ & 251.28 & 198-199 & 66.2 & 0.78 \\
\hline $3 b$ & $\mathrm{CH}_{3}-$ & $\mathrm{C}_{16} \mathrm{H}_{15} \mathrm{~N}_{3} \mathrm{O}$ & 265.31 & $180-182$ & 62.4 & 0.69 \\
\hline $3 c$ & $\mathrm{ClCH}_{2-}^{-}$ & $\mathrm{C}_{16} \mathrm{H}_{14} \mathrm{CIN}_{3} \mathrm{O}$ & 299.75 & $190-191$ & 76.5 & 0.59 \\
\hline $3 d$ & $\mathrm{C}_{2} \mathrm{H}_{5}^{-}$ & $\mathrm{C}_{17} \mathrm{H}_{17} \mathrm{~N}_{3} \mathrm{O}$ & 279.34 & $210-212$ & 64.2 & 0.64 \\
\hline $3 e$ & $\mathrm{C}_{3} \mathrm{H}_{8}^{-}$ & $\mathrm{C}_{18} \mathrm{H}_{19} \mathrm{~N}_{3} \mathrm{O}$ & 293.36 & $208-210$ & 62.5 & 0.77 \\
\hline $3 f$ & $\mathrm{C}_{4} \mathrm{H}_{10^{-}}$ & $\mathrm{C}_{19} \mathrm{H}_{21} \mathrm{~N}_{3} \mathrm{O}$ & 307.39 & $212-214$ & 60.6 & 0.91 \\
\hline $3 g$ & $\mathrm{NH}_{2}^{-}$ & $\mathrm{C}_{15} \mathrm{H}_{14} \mathrm{~N}_{4} \mathrm{O}$ & 266.30 & $205-206$ & 58.4 & 0.90 \\
\hline $3 \mathrm{~h}$ & $\mathrm{GH}^{\mathrm{H}}$ & $\mathrm{C}_{17} \mathrm{H}_{17} \mathrm{~N}_{3} \mathrm{O}_{2}$ & 295.34 & $190-192$ & 56.2 & 0.65 \\
\hline $3 i$ & $\mathrm{SH}-$ & $\mathrm{C}_{15} \mathrm{H}_{13} \mathrm{~N}_{3} \mathrm{OS}$ & 283.35 & $170-172$ & 52.7 & 0.91 \\
\hline $3 \mathrm{j}$ & $\mathrm{SHCH}_{2}-$ & $\mathrm{C}_{16} \mathrm{H}_{15} \mathrm{~N}_{3} \mathrm{OS}$ & 297.38 & $212-214$ & 51.8 & 0.84 \\
\hline $3 k$ & $\mathrm{C}_{6} \mathrm{H}_{5}^{-}$ & $\mathrm{C}_{21} \mathrm{H}_{17} \mathrm{~N}_{3} \mathrm{O}$ & 327.38 & $195-197$ & 80.4 & 0.95 \\
\hline 31 & $\left(2-\mathrm{N}-\mathrm{C}_{5} \mathrm{H}_{4}\right)-$ & $\mathrm{C}_{20} \mathrm{H}_{16} \mathrm{~N}_{4} \mathrm{O}$ & 328.37 & $218-220$ & 72.5 & 0.77 \\
\hline $3 m$ & $\left(3-\mathrm{N}-\mathrm{C}_{5} \mathrm{H}_{4}\right)-$ & $\mathrm{C}_{20} \mathrm{H}_{16} \mathrm{~N}_{4} \mathrm{O}$ & 328.37 & $205-206$ & 79.7 & 0.85 \\
\hline $3 n$ & $\left(2-\mathrm{OH}-\mathrm{C}_{6} \mathrm{H}_{4}\right)-$ & $\mathrm{C}_{21} \mathrm{H}_{17} \mathrm{~N}_{3} \mathrm{O}_{2}$ & 343.38 & $180-182$ & 75.3 & 0.84 \\
\hline 30 & $\left(2-\mathrm{Cl}-\mathrm{C}_{6} \mathrm{H}_{4}\right)-$ & $\mathrm{C}_{21} \mathrm{H}_{16} \mathrm{ClN}_{3} \mathrm{O}$ & 361.82 & $190-191$ & 80.0 & 0.87 \\
\hline $3 p$ & $\left(3-\mathrm{Cl}-\mathrm{C}_{6} \mathrm{H}_{4}\right)-$ & $\mathrm{C}_{21} \mathrm{H}_{16} \mathrm{CIN}_{3} \mathrm{O}$ & 361.82 & $200-202$ & 71.3 & 0.86 \\
\hline $3 q$ & $\left(4-\mathrm{Cl}-\mathrm{C}_{6} \mathrm{H}_{4}\right)-$ & $\mathrm{C}_{21} \mathrm{H}_{16} \mathrm{ClN}_{3} \mathrm{O}$ & 361.82 & $195-196$ & 73.6 & 0.75 \\
\hline $3 r$ & $\left(2-\mathrm{Br}-\mathrm{C}_{6} \mathrm{H}_{4}\right)-$ & $\mathrm{C}_{21} \mathrm{H}_{16} \mathrm{BrN}_{3} \mathrm{O}$ & 406.28 & $190-192$ & 75.4 & 0.81 \\
\hline $3 s$ & $\left(3-\mathrm{Br}-\mathrm{C}_{6} \mathrm{H}_{4}\right)-$ & $\mathrm{C}_{21} \mathrm{H}_{16} \mathrm{BrN}_{3} \mathrm{O}$ & 406.28 & 192-194 & 76.7 & 0.80 \\
\hline $3 t$ & $\left(4-\mathrm{Br}-\mathrm{C}_{6} \mathrm{H}_{4}\right)-$ & $\mathrm{C}_{21} \mathrm{H}_{16} \mathrm{BrN}_{3} \mathrm{O}$ & 406.28 & $195-196$ & 78.6 & 0.77 \\
\hline $3 u$ & $\left(4-\mathrm{NO}_{2}-\mathrm{C}_{6} \mathrm{H}_{4}\right)-$ & $\mathrm{C}_{21} \mathrm{H}_{16} \mathrm{~N}_{4} \mathrm{O}_{3}$ & 372.38 & $180-183$ & 79.2 & 0.64 \\
\hline $3 v$ & $\left(-\mathrm{C}_{6} \mathrm{H}_{5}-\mathrm{CH}_{2}\right)-$ & $\mathrm{C}_{22} \mathrm{H}_{19} \mathrm{~N}_{3} \mathrm{O}$ & 341.41 & $170-172$ & 64.6 & 0.70 \\
\hline $3 w$ & $\left(4-\mathrm{F}-\mathrm{C}_{6} \mathrm{H}_{4}\right)-$ & $\mathrm{C}_{21} \mathrm{H}_{16} \mathrm{FN}_{3} \mathrm{O}$ & 345.37 & $230-231$ & 72.7 & 0.76 \\
\hline $3 x$ & $\left(2-\mathrm{NH}_{2}-\mathrm{C}_{6} \mathrm{H}_{4}\right)-$ & $\mathrm{C}_{21} \mathrm{H}_{18} \mathrm{~N}_{4} \mathrm{O}$ & 342.39 & $206-208$ & 78.5 & 0.88 \\
\hline
\end{tabular}

Stationary phase: Silica gel G, Mobile phase for TLC : chloroform: methanol ( $9.5: 0.5)$

7.4-8.1 (m, 9H, ArH), $4.92\left(\mathrm{~s}, 2 \mathrm{H}, \mathrm{NCH}_{2} \mathrm{~N}\right), 9.0$ (s, 1H, $\mathrm{NH}), 5.19\left(\mathrm{~s}, 2 \mathrm{H}, \mathrm{CH}_{2}\right)$. Anal Calcd for $\mathrm{C}_{16} \mathrm{H}_{14} \mathrm{ClN}_{3} \mathrm{O}: \mathrm{C}$ 64.11, H 4.71, N 14.02; found C 65.06, H 4.41, N 13.9. MS: $\mathrm{m} / \mathrm{z}=300.2(\mathrm{M}+1)$.

\section{N-(2-ethyl-benzimidazol-1-yl methyl)-benzamide (3d)}

White crystals, mp $210-212^{\circ} \mathrm{C}, \mathrm{IR}(\mathrm{KBr}, \mathrm{cm}-1) 3308$ (N-H), $1486(\mathrm{C}=\mathrm{N}), 3019$ C-H $\left(\mathrm{CH}_{2}\right), 2967$ (C-H, Ar.) $1526(\mathrm{C}=\mathrm{C}), 1634(\mathrm{C}=\mathrm{O})$, 675-870 (CH bend.Ar). 1HNMR (400 MHz, DMSO-d 6 , $8 \mathrm{ppm}), 7.3-7.9(\mathrm{~m}, 9 \mathrm{H}$, $\mathrm{ArH}), 4.99$ (s, $2 \mathrm{H}, \mathrm{NCH}_{2} \mathrm{~N}$ ), 8.8 (s, 1H, NH), 3.27 (q, $\left.2 \mathrm{H}, \mathrm{CH}_{2}, \mathrm{~J}=7.6 \mathrm{~Hz}\right), 1.56\left(\mathrm{t}, 3 \mathrm{H}, \mathrm{CH}_{3}, \mathrm{~J}=7.6 \mathrm{~Hz}\right.$ ). Anal Calcd for $\mathrm{C}_{17} \mathrm{H}_{17} \mathrm{~N}_{3} \mathrm{O}$ : C 73.10, H 6.13 N 15.04; found $\mathrm{C}$ 71.06, H 5.98, N 14.9. MS: $\mathrm{m} / \mathrm{z}=279.2(\mathrm{M}+1)$.

\section{N-(2-propyl-benzimidazol-1-yl methyl)-benzamide (3e)}

White crystals, $\mathrm{mp} 208-210^{\circ} \mathrm{C}, \mathrm{IR}(\mathrm{KBr}, \mathrm{cm}-1) 3308(\mathrm{~N}-\mathrm{H})$, $1487(\mathrm{C}=\mathrm{N}), 3056$ C-H $\left(\mathrm{CH}_{2}\right), 2967$ (C-H,Ar.) 1527
$(\mathrm{C}=\mathrm{C}), 1634(\mathrm{C}=\mathrm{O})$, 675-870 (CH bend.Ar). 1HNMR (400MHz, DMSO-d 6 , 8ppm), 7.40-8.12 (m, 9H, ArH), 4.93 (s, 2H, NCH$\left.{ }_{2} \mathrm{~N}\right), 8.9$ (s, 1H, NH), 2.96 (t, 2H, $\mathrm{CH}_{2}$, $\mathrm{J}=7.6 \mathrm{~Hz}$ ), 1.93 (sextet, $\left.2 \mathrm{H}, \mathrm{CH}_{2}, \mathrm{~J}=7.6 \mathrm{~Hz}\right), 1.0$ (t, 3H, $\mathrm{CH}_{3}, \mathrm{~J}=7.2 \mathrm{~Hz}$ ). Anal Calcd for $\mathrm{C}_{18} \mathrm{H}_{19} \mathrm{~N}_{3} \mathrm{O}: \mathrm{C} 73.69, \mathrm{H}$ 6.53, N 14.32; found C 75.4, H 5.96 N 14.15.

\section{N-(2-butyl-benzimidazol-1-yl methyl)-benzamide (3f)}

White crystals, mp $212-214^{\circ} \mathrm{C}, \mathrm{IR}(\mathrm{KBr}, \mathrm{cm}-1) 3307$ (N-H), $1486(\mathrm{C}=\mathrm{N}), 3055 \mathrm{C}-\mathrm{H}\left(\mathrm{CH}_{2}\right), 2966(\mathrm{C}-\mathrm{H}, \mathrm{Ar}$. 1527 ( $\mathrm{C}=\mathrm{C}), 1635$ ( $\mathrm{C}=\mathrm{O})$, 675-870 ( $\mathrm{CH}$ bend.Ar). $1 \mathrm{H}$ NMR (400MHz, DMSO-d $\left.{ }_{6}, 8 p p m\right), 7.18-8.06$ (m, 9H, ArH), 4.97 (s, 2H, $\mathrm{NCH}_{2} \mathrm{~N}$ ), 8.84 (s, 1H, NH), 0.93 $\left(\mathrm{t}, 3 \mathrm{H}, \mathrm{CH}_{3}, \mathrm{~J}=7.2 \mathrm{~Hz}\right.$ ), 1.40 (sextet, $2 \mathrm{H}, \mathrm{CH}_{2}, \mathrm{~J}=7.6 \mathrm{~Hz}$ ), $1.82\left(\mathrm{p}, 2 \mathrm{H}, \mathrm{CH}_{2}, \mathrm{~J}=2.16 \mathrm{~Hz}\right), 2.93\left(\mathrm{t}, 2 \mathrm{H}, \mathrm{CH}_{2}, \mathrm{~J}=7.6\right.$ $\mathrm{Hz}$ ). Anal Calcd for $\mathrm{C}_{19} \mathrm{H}_{21} \mathrm{~N}_{3} \mathrm{O}: \mathrm{C}$ 74.24, $\mathrm{H}$ 6.89, N 13.67; found C 69.4, H 6.59, N 12.43. 


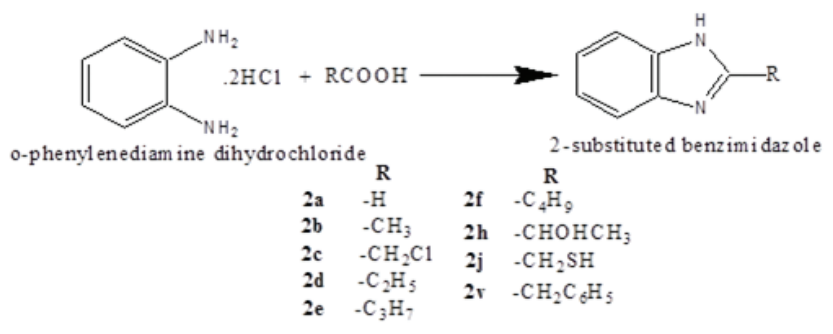

Scheme 1a: Synthesis of 2-substituted benzimidazoles using o-phenylene diamine dihydrochloride.

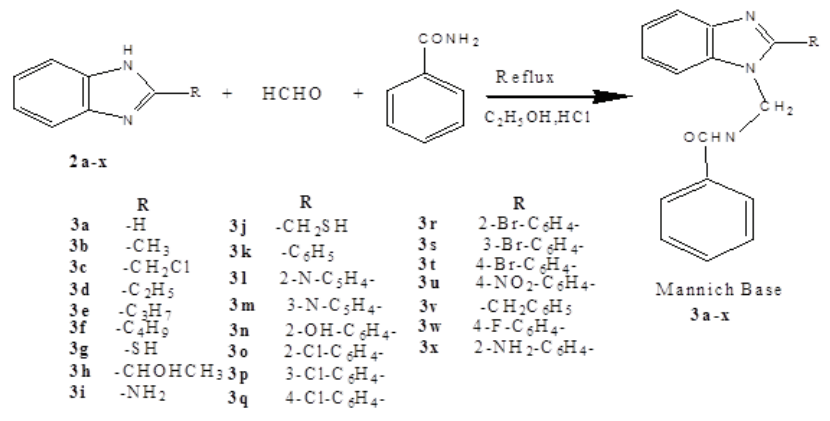

Scheme 2: Synthesis of mannich bases from 2-substituted benzimidazoles.

N-(2-amino-benzimidazol-1-yl methyl)-benzamide (3g)

Yellow crystals; mp. 205-206 ${ }^{\circ} \mathrm{C}, \mathrm{IR}(\mathrm{KBr}, \mathrm{cm}-1) 3305$ $(\mathrm{N}-\mathrm{H}), 1484(\mathrm{C}=\mathrm{N}), 3165 \mathrm{C}-\mathrm{H}\left(\mathrm{CH}_{2}\right), 3060(\mathrm{C}-\mathrm{H}, \mathrm{Ar}$. $1529(\mathrm{C}=\mathrm{C}), 1638(\mathrm{C}=\mathrm{O}), 675-870$ (CH bend.Ar). $1 \mathrm{H}$ NMR (400MHz, DMSO-d $\left.\mathrm{d}_{6}, 8 \mathrm{ppm}\right), 7.21-7.96(\mathrm{~m}, 9 \mathrm{H}$, $\mathrm{ArH}), 7.97$ (s,1H, $\mathrm{NH}_{2}$ ), 4.90 (s, $\left.2 \mathrm{H}, \mathrm{NCH}_{2} \mathrm{~N}\right), 9.03(\mathrm{~s}$, $1 \mathrm{H}, \mathrm{NH}), 9.6\left(\mathrm{~s}, 1 \mathrm{H}, \mathrm{NH}_{2}\right.$ ). Anal Calcd for $\mathrm{C}_{15} \mathrm{H}_{14} \mathrm{~N}_{4} \mathrm{O}$ : C 67.65, H 5.30 , N 21.04; found C 65.3, H 4.92, N 20.3.

\section{N-2-[(1-hydroxy-ethyl)-benzimidazol-1-ylmethyl]-} benzamide (3h)

White crystals, mp $190-192^{\circ} \mathrm{C}$ IR $(\mathrm{KBr}, \mathrm{cm}-1) 3308$ (N-H), $1487(\mathrm{C}=\mathrm{N}), 3056 \mathrm{C}-\mathrm{H}\left(\mathrm{CH}_{2}\right), 2967$ (C-H, Ar.) $1527(\mathrm{C}=\mathrm{C}), 1634(\mathrm{C}=\mathrm{O}), 675-870$ ( $\mathrm{CH}$ bend.Ar). 1H NMR (400MHz, DMSO-d , $8 \mathrm{ppm}), 7.39-7.92$ (m, 9H, ArH), 4.90 (s, 2H, $\left.\mathrm{NCH}_{2} \mathrm{~N}\right), 8.97$ (s, 1H, NH), 5.70 (br, s, $1 \mathrm{H}, \mathrm{OH}), 1.59\left(\mathrm{~d}, 3 \mathrm{H} \mathrm{CH}_{3}, \mathrm{~J}=6.4 \mathrm{~Hz}\right), 5.03(1 \mathrm{H}, \mathrm{q}$, $\mathrm{CH}, \mathrm{J}=6.8 \mathrm{~Hz}$ ). Anal Calcd for $\mathrm{C}_{17} \mathrm{H}_{17} \mathrm{~N}_{3} \mathrm{O}_{2}: \mathrm{C} 69.14$, H 5.80, N 14.23 ; found C 68.3, H 5.42, N 13.92.

\section{N-(2-mercapto-benzimidazol-1-ylmethyl)-benzamide (3i)}

White crystals, mp $270-272^{\circ} \mathrm{C}, \mathrm{IR}(\mathrm{KBr}, \mathrm{cm}-1) 3305$ $(\mathrm{N}-\mathrm{H}), 1378(\mathrm{C}=\mathrm{N}), 3178 \mathrm{C}-\mathrm{H}\left(\mathrm{CH}_{2}\right), 3056(\mathrm{C}-\mathrm{H}, \mathrm{Ar}$. $1526(\mathrm{C}=\mathrm{C}), 1635(\mathrm{C}=\mathrm{O}), 675-870$ (CH bend.Ar).1H NMR (400MHz, DMSO-d , $8 \mathrm{ppm}), 7.26-7.95$ (m, 9H, ArH), 4.95 (s, 2H, $\left.\mathrm{NCH}_{2} \mathrm{~N}\right), 8.86$ (s, 1H, NH), 3.4
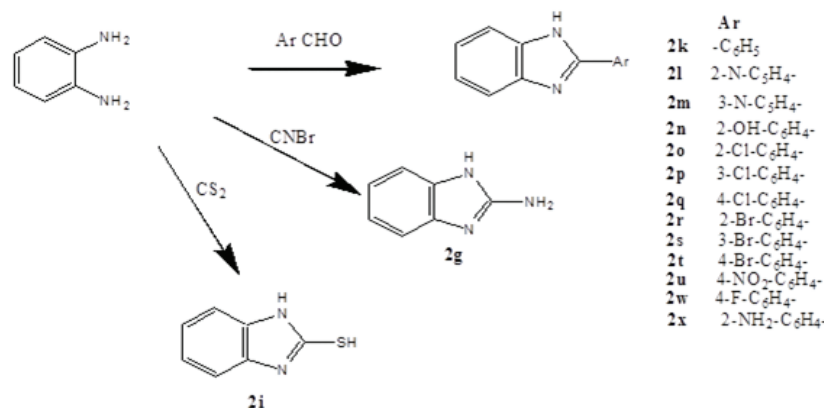

Scheme 1b: Synthesis of 2-substituted benzimidazoles using o-phenylene diamine.

(s, $1 \mathrm{H}, \mathrm{SH})$. Anal Calcd for $\mathrm{C}_{15} \mathrm{H}_{13} \mathrm{~N}_{3} \mathrm{OS}: \mathrm{C} 63.58, \mathrm{H} 4.62$, N 14.83; found C 62.3, H 4.42, N 14.62.

N-(2-mercaptomethyl-benzimidazol-1-ylmethyl)benzamide (3j)

White crystals, mp $212-214^{\circ} \mathrm{C}, \mathrm{IR}(\mathrm{KBr}, \mathrm{cm}-1) 3308$ $(\mathrm{N}-\mathrm{H}), 1487(\mathrm{C}=\mathrm{N}), 3056 \mathrm{C}-\mathrm{H}\left(\mathrm{CH}_{2}\right), 2966(\mathrm{C}-\mathrm{H}, \mathrm{Ar}$. $1526(\mathrm{C}=\mathrm{C}), 1634(\mathrm{C}=\mathrm{O})$, 675-870 (CH bend.Ar). $1 \mathrm{H}$ NMR (400MHz, DMSO-d, 6 , $p p m), 7.4-8.12$ (m, 9H, $\mathrm{ArH}), 4.92$ (s, 2H, $\left.\mathrm{NCH}_{2} \mathrm{~N}\right), 8.98$ (s, 1H, NH), 3.39 (s, $1 \mathrm{H}, \mathrm{SH}), 4.18\left(\mathrm{~s}, 2 \mathrm{H}, \mathrm{CH}_{2}\right)$. Anal Calcd for $\mathrm{C}_{16} \mathrm{H}_{15} \mathrm{~N}_{3}$ OS : C 64.62, H 5.08, N 14.13; found C 63.3, H 4.96, N 13.92. MS: $\mathrm{m} / \mathrm{z}=298.2(\mathrm{M}+1)$.

\section{N-(2-phenyl-benzimidazol-1-ylmethyl)-benzamide (3k)}

Brown crystals; mp. $195-197{ }^{\circ} \mathrm{C}, \mathrm{IR}(\mathrm{KBr}, \mathrm{cm}-1) 3308$ $(\mathrm{N}-\mathrm{H}), 1488(\mathrm{C}=\mathrm{N}), 3056 \mathrm{C}-\mathrm{H}\left(\mathrm{CH}_{2}\right), 2965$ (C-H, Ar.) $1526(\mathrm{C}=\mathrm{C}), 1634(\mathrm{C}=\mathrm{O}), 675-870$ ( $\mathrm{CH}$ bend.Ar). $1 \mathrm{H}$ NMR $\left(400 \mathrm{MHz}\right.$, DMSO-d $\left.\mathrm{d}_{6}, 8 \mathrm{ppm}\right), 7.4-8.32(\mathrm{~m}, 14 \mathrm{H}$, $\mathrm{ArH}), 4.93\left(\mathrm{~s}, 2 \mathrm{H}, \mathrm{NCH}_{2} \mathrm{~N}\right), 9.0(\mathrm{~s}, 1 \mathrm{H}, \mathrm{NH})$. Anal Calcd for $\mathrm{C}_{21} \mathrm{H}_{17} \mathrm{~N}_{3} \mathrm{O}$ : C 77.04, H 5.23, N 12.84 ; found C 76.3, H 5.20, N 12.72. MS: $\mathrm{m} / \mathrm{z}=329.2(\mathrm{M}+1)$.

\section{N-(2-pyridin-2-yl-benzimidazol-1-ylmethyl)-benzamide (3l)}

Off white crystals; mp. $218-220^{\circ} \mathrm{C}, \mathrm{IR}(\mathrm{KBr}, \mathrm{cm}-1) 3308$ $(\mathrm{N}-\mathrm{H}), 1447(\mathrm{C}=\mathrm{N}), 3056 \mathrm{C}-\mathrm{H}\left(\mathrm{CH}_{2}\right), 2967$ (C-H, Ar.) $1527(\mathrm{C}=\mathrm{C}), 1634(\mathrm{C}=\mathrm{O}), 675-870$ (CH bend.Ar). $1 \mathrm{H}$ NMR (400MHz, DMSO-d, $8 p p m), 7.39-7.91(\mathrm{~m}, 13 \mathrm{H}$, ArH), $4.9\left(\mathrm{~s}, 2 \mathrm{H}, \mathrm{NCH}_{2} \mathrm{~N}\right), 8.8(\mathrm{~s}, 1 \mathrm{H}, \mathrm{NH})$. Anal Calcd for $\mathrm{C}_{20} \mathrm{H}_{16} \mathrm{~N}_{4} \mathrm{O}: \mathrm{C} 73.15, \mathrm{H}$ 4.91, N 17.06 ; found $\mathrm{C}$ 73.3, H 4.20, N 16.72 .

\section{N-(2-pyridin-3-yl-benzimidazol-1-ylmethyl)-benzamide (3m)}

Yellow crystals; mp. $205-206^{\circ} \mathrm{C}, \mathrm{IR}(\mathrm{KBr}, \mathrm{cm}-1) 3308$ $(\mathrm{N}-\mathrm{H}), 1486(\mathrm{C}=\mathrm{N}), 3053 \mathrm{C}-\mathrm{H}\left(\mathrm{CH}_{2}\right), 2966$ (C-H,Ar.), $1527(\mathrm{C}=\mathrm{C}), 1635$ ( $\mathrm{C}=\mathrm{O}), 675-870$ (CH bend.Ar). $1 \mathrm{H}$ NMR $\left(400 \mathrm{MHz}\right.$, DMSO-d $\left.{ }_{6}, \delta \mathrm{ppm}\right), 7.2-8.96(\mathrm{~m}, 13 \mathrm{H}$, ArH), 4.9 (s, 2H, $\left.\mathrm{NCH}_{2} \mathrm{~N}\right), 9.5$ (s, 1H, NH). Anal Calcd for $\mathrm{C}_{20} \mathrm{H}_{16} \mathrm{~N}_{4} \mathrm{O}$ : C 73.15, H 4.9, N 17.6 ; found $\mathrm{C}$ 72.06, H 5.6, N 16.92. MS: $\mathrm{m} / \mathrm{z}=329.3(\mathrm{M}+1)$. 
N-[2-(2-hydroxy-phenyl)-benzimidazol-1-ylmethyl]benzamide (3n)

White crystals; mp. $180-182^{\circ} \mathrm{C}, \mathrm{IR}(\mathrm{KBr}, \mathrm{cm}-1) 3310$ (N-H), 1491 (C=N), 3057 C-H $\left(\mathrm{CH}_{2}\right), 2964$ (C-H, Ar.) 1527 (C=C), 1633 (C=O), 675-870 (CH bend.Ar). $1 \mathrm{H}$ NMR (400MHz, DMSO-d, $\delta$ ppm), 7.04-8.18 (m, 13H, ArH), 4.91 (s, 2H, $\mathrm{NCH}_{2} \mathrm{~N}$ ), 9.0 (s, 1H, NH), 13.6 (br,s, $1 \mathrm{H}, \mathrm{OH})$. Anal Calcd for $\mathrm{C}_{21} \mathrm{H}_{17} \mathrm{~N}_{3} \mathrm{O}_{2}$ : C 73.45, H 4.99, $\mathrm{N} 12.24$; found $\mathrm{C} 72.06, \mathrm{H} 4.63, \mathrm{~N} \mathrm{11.92.} \mathrm{MS:} \mathrm{m} / \mathrm{z}=$ $344.8(\mathrm{M}+1)$.

N-[2-(2-chloro-phenyl)-benzimidazol-1-ylmethyl]benzamide (30)

White crystals; mp. $190-191^{\circ} \mathrm{C}, \mathrm{IR}(\mathrm{KBr}, \mathrm{cm}-1) 3307$ (N-H), $1485(\mathrm{C}=\mathrm{N}), 3048 \mathrm{C}-\mathrm{H}\left(\mathrm{CH}_{2}\right), 2963$ (C-H, Ar.) $1526(\mathrm{C}=\mathrm{C}), 1634$ (C=O), 770 (C-Cl), 675-870 (CH bend.Ar). $1 H$ NMR (400MHz, DMSO-d, $8 p p m), 7.41-$ 8.9 (m, 13H, ArH), 4.91 (s, 2H, $\left.\mathrm{NCH}_{2} \mathrm{~N}\right), 9.01(\mathrm{~s}, 1 \mathrm{H}$, $\mathrm{NH})$. Anal Calcd for $\mathrm{C}_{21} \mathrm{H}_{16} \mathrm{ClN}_{3} \mathrm{O}: \mathrm{C}$ 69.71, $\mathrm{H} 4.46, \mathrm{~N}$ 11.61; found C 68.5, H 4.31, N 10.2. MS: $\mathrm{m} / \mathrm{z}=362.2$ $(\mathrm{M}+1), 363(\mathrm{M}+2)$.

N-[2-(3-chloro-phenyl)-benzimidazol-1-ylmethyl]benzamide (3p)

White crystals; mp. $200-202^{\circ} \mathrm{C}, \mathrm{IR}(\mathrm{KBr}, \mathrm{cm}-1) 3309$ (N-H), 1485 (C=N), 3034 C-H $\left(\mathrm{CH}_{2}\right), 2953$ (C-H,Ar.) 1527 (C=C), 1633 (C=O), 765 (C-Cl), 675-870 (CH bend.Ar). $1 H$ NMR (400MHz, DMSO-d, $8 p p m), 7.46-$ 8.7 (m, 13H, ArH) 4.98 (s, 2H, $\left.\mathrm{NCH}_{2} \mathrm{~N}\right), 8.9$ (s, 1H, $\mathrm{NH})$. Anal Calcd for $\mathrm{C}_{21} \mathrm{H}_{16} \mathrm{ClN}_{3} \mathrm{O}: \mathrm{C} 69.71, \mathrm{H} 4.46, \mathrm{~N}$ 11.61; found $\mathrm{C}$ 68.5, $\mathrm{H} 4.81, \mathrm{~N} 11.2$.

N-[2-(4-chloro-phenyl)-benzimidazol-1-ylmethyl]benzamide (3q)

White crystals; mp. $195-196^{\circ} \mathrm{C}, \mathrm{IR}$ (KBr, cm-1) 3307 (N-H), 1485 (C=N), 3045 C-H(CH$) 2952$ (C-H, Ar.) $1525(\mathrm{C}=\mathrm{C}), \quad 1635(\mathrm{C}=\mathrm{O}), \quad 764(\mathrm{C}-\mathrm{Cl}) \quad 675-870(\mathrm{CH}$ bend.Ar). $1 H$ NMR (400MHz, DMSO-d , $8 \mathrm{ppm}), 7.35-$ 8.6 (m, 13H, ArH), 4.96 (s, 2H, $\left.\mathrm{NCH}_{2} \mathrm{~N}\right), 8.90$ (s, 1H, $\mathrm{NH})$. Anal Calcd for $\mathrm{C}_{21} \mathrm{H}_{16} \mathrm{ClN}_{3} \mathrm{O}: \mathrm{C} 69.71, \mathrm{H} 4.46, \mathrm{~N}$ 11.61 ; found C 68.52, H 4.31, N 10.8. MS: $\mathrm{m} / \mathrm{z}=363.9$ $(\mathrm{M}+2)$.

\section{N-[2-(2-bromo-phenyl)-benzimidazol-1-ylmethyl]- benzamide (3r)}

White crystals; mp. $190-192^{\circ} \mathrm{C}, \mathrm{IR}(\mathrm{KBr}, \mathrm{cm}-1) 3309$ (N-H), $1488(\mathrm{C}=\mathrm{N}), 3056 \mathrm{C}-\mathrm{H}\left(\mathrm{CH}_{2}\right), 2967$ (C-H, Ar.) 1527 (C=C), 1635 (C=O), 869 (C-Br), 675-870 (CH bend.Ar). $1 H$ NMR (400MHz, DMSO-d,$\delta p p m), 7.4-$ 8.1 (m, 13H, $\mathrm{ArH}), 4.91\left(\mathrm{~s}, 2 \mathrm{H}, \mathrm{NCH}_{2} \mathrm{~N}\right), 8.9$ (s, 1H, $\mathrm{NH})$. Anal Calcd for $\mathrm{C}_{21} \mathrm{H}_{16} \mathrm{BrN}_{3} \mathrm{O}: \mathrm{C} 62.08, \mathrm{H}$ 3.97, N 10.34; found C 63.3, H 2.98, N 9.43. MS: $\mathrm{m} / \mathrm{z}=406.2$ $(\mathrm{M}+1), 407.3(\mathrm{M}+2)$.
N-[2-(3-bromo-phenyl)-benzimidazol-1-ylmethyl]benzamide (3s)

White crystals; mp. $192-194^{\circ} \mathrm{C}$, IR (KBr, cm-1) 3307 (N-H), $1487(\mathrm{C}=\mathrm{N}), 3034 \mathrm{C}-\mathrm{H}\left(\mathrm{CH}_{2}\right), 2966$ (C-H, Ar.) 1525 (C=C), 1634 (C=O), 865 (C-Br), 675-870 (CH

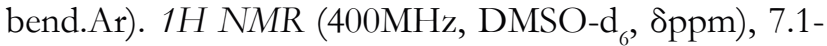
8.7 (m, 13H, ArH), 4.98(s, 2H, $\left.\mathrm{NCH}_{2} \mathrm{~N}\right), 9.0$ (s, 1H, $\mathrm{NH}$ ). Anal Calcd for $\mathrm{C}_{21} \mathrm{H}_{16} \mathrm{BrN}_{3} \mathrm{O}: \mathrm{C} 62.08, \mathrm{H}$ 3.97, $\mathrm{N}$ 10.34 ; found C 61.3, H 3.90, N 9.49.

N-[2-(4-bromo-phenyl)-benzimidazol-1-ylmethyl]benzamide (3t)

Off white crystals; mp. $195-196^{\circ} \mathrm{C}, \mathrm{IR}(\mathrm{KBr}, \mathrm{cm}-1) 3308$ (N-H), $1486(\mathrm{C}=\mathrm{N}), 3056$ C-H $\left(\mathrm{CH}_{2}\right), 2967$ (C-H, Ar.) 1527 (C=C), 1635 (C=O), 865(C-Br), 675-870 (CH bend.Ar). $1 H$ NMR (400MHz, DMSO-d, $8 p p m), 7.2-$ 8.6 (m, 13H, ArH), 4.97 (s, 2H, $\mathrm{NCH}_{2} \mathrm{~N}$ ), 8.89 (s, 1H, $\mathrm{NH}$ ). Anal Calcd for $\mathrm{C}_{21} \mathrm{H}_{16} \mathrm{BrN}_{3} \mathrm{O}: \mathrm{C}$ 62.08, H 3.97, N 10.34 ; found C 61.9, H 2.96, N 9.82.

N-[2-(4-nitro-phenyl)-benzimidazol-1-ylmethyl]benzamide (3u)

Yellow crystals; mp. $180-183^{\circ} \mathrm{C}, \mathrm{IR}(\mathrm{KBr}$; cm-1) 3308 $(\mathrm{N}-\mathrm{H}), 1463(\mathrm{C}=\mathrm{N}), 3058 \mathrm{C}-\mathrm{H}\left(\mathrm{CH}_{2}\right), 2966$ (C-H, Ar.) $1524(\mathrm{C}=\mathrm{C}), 1634(\mathrm{C}=\mathrm{O}), 747\left(\mathrm{C}-\mathrm{NO}_{2}\right), 675-870(\mathrm{CH}$ bend.Ar). $1 H$ NMR (400MHz, DMSO-d , $8 \mathrm{ppm}), 7.24-$ 8.46 (m, 13H, ArH), 4.94 (s, 2H, $\left.\mathrm{NCH}_{2} \mathrm{~N}\right), 8.90$ (s, 1H, $\mathrm{NH}$ ). Anal Calcd for $\mathrm{C}_{21} \mathrm{H}_{16} \mathrm{~N}_{4} \mathrm{O}_{3}$ : C 67.73, H 4.33, N 15.05; found C 66.3, H 4.28, N 14.96. MS: $\mathrm{m} / \mathrm{z}=373.7$ $(\mathrm{M}+1)$.

\section{N-(2-benzyl-benzimidazol-1-ylmethyl)-benzamide (3v)}

White crystals; mp. $170-172^{\circ} \mathrm{C}, \mathrm{IR}(\mathrm{KBr}, \mathrm{cm}-1) 3308$ (N-H), 1487 (C=N), 3054 C-H $\left(\mathrm{CH}_{2}\right), 2966$ (C-H, Ar.) $1525(\mathrm{C}=\mathrm{C}), 1634(\mathrm{C}=\mathrm{O}), 675-870$ (CH bend.Ar). $1 \mathrm{H}$ NMR (400MHz, DMSO-d, $8 \mathrm{ppm}), 7.24-7.93$ (m, 14H, $\mathrm{ArH}), 4.93\left(\mathrm{~s}, 2 \mathrm{H}, \mathrm{NCH}_{2} \mathrm{~N}\right), 8.62$ (s, 1H, NH), 4.53 (s, $2 \mathrm{H}, \mathrm{CH}_{2}$ ). Anal Calcd for $\mathrm{C}_{22} \mathrm{H}_{19} \mathrm{~N}_{3} \mathrm{O}: \mathrm{C} 77.40$, H 5.61, $\mathrm{N}$ 12.31; found C 76.3, H 5.49, N 11.94.

\section{N-[2-(4-flouro-phenyl)-benzimidazol-1-ylmethyl]-} benzamide $(3 w)$

White crystals; mp. $230-231^{\circ} \mathrm{C}, \mathrm{IR}$ ( $\left.\mathrm{KBr}, \mathrm{cm}-1\right) 3310$ (N-H), $1458(\mathrm{C}=\mathrm{N}), 3055 \mathrm{C}-\mathrm{H}\left(\mathrm{CH}_{2}\right), 2927$ (C-H, Ar.) 1525 (C=C), 1634 (C=O), 848 (C-F), 675-870 (CH bend. Ar). 1H NMR (400MHz, DMSO-d, $8 \mathrm{ppm}), 7.4-8.48$ (m, 13H, ArH), 4.90 (s, 2H, $\mathrm{NCH}_{2} \mathrm{~N}$ ), 9.03 (s, 1H, NH). Anal Calcd for $\mathrm{C}_{21} \mathrm{H}_{16} \mathrm{FN}_{3} \mathrm{O}$ : C 73.03, $\mathrm{H}$ 4.67, $\mathrm{N} \mathrm{12.17}$; found C 72.7, H 3.98, N 11.82. MS: $\mathrm{m} / \mathrm{z}=346.3(\mathrm{M}+1)$.

\section{N-[2-(2-amino-phenyl)-benzimidazol-1-ylmethyl]- benzamide $(3 x)$}

Yellow crystals; mp. $206-208^{\circ} \mathrm{C}, \mathrm{IR}$ (KBr, cm-1) 3310 (N-H), $1486(\mathrm{C}=\mathrm{N}), 3086 \mathrm{C}-\mathrm{H}\left(\mathrm{CH}_{2}\right), 2959$ (C-H, Ar.) 
$1525(\mathrm{C}=\mathrm{C}), 1634(\mathrm{C}=\mathrm{O}), 675-870$ (CH bend.Ar). $1 \mathrm{H}$ NMR (400MHz, DMSO-d,, $8 \mathrm{ppm}), 6.52-7.95$ (m, 13H, ArH), 4.98 (s, 2H, $\mathrm{NCH}_{2} \mathrm{~N}$ ), 8.79 (s, $\left.1 \mathrm{H}, \mathrm{NH}\right), 4.0$ (s, $2 \mathrm{H}, \mathrm{NH}_{2}$ ). Anal Calcd for $\mathrm{C}_{21} \mathrm{H}_{18} \mathrm{~N}_{4} \mathrm{O}$ : C 73.67, H 5.30, $\mathrm{N}$ 16.36; found C 71.9, H 4.96, N 15.82. MS: $\mathrm{m} / \mathrm{z}=$ $343.5(\mathrm{M}+1)$.

\section{Antimicrobial Evaluation}

The synthesized compounds were screened for their in vitro antimicrobial activity against two gram positive bacterial strains: Staphylococcus aureus (MTCC 96), Bacillus subtilis (MTCC 121), two gram negative bacterial strains: Escherichia coli (MTCC 40), Pseudomonas aeruginosa (MTCC 2453), and two fungal strains: Candida albicans (MTCC 183) and Aspergillus niger (MTCC 404). The serial dilution technique was used for evaluation of antimicrobial activity. Stock solution of synthesized compounds was prepared in dimethyl sulphoxide $(100 \mu \mathrm{g} / \mathrm{ml})$. Nutrient broth (I.P.) and sabouraud dextrose broth media (I.P.) ${ }^{29}$ were used for bacteria and fungi respectively. Ciprofloxacin and clotrimazole were used as standard drugs for antibacterial activity and antifungal activity respectively. Sterlized media $(1 \mathrm{ml})$ was transferred into sterile test tubes. Stock solution $(100 \mu \mathrm{g} / \mathrm{ml})(1 \mathrm{ml})$ was put in one tube and serially diluted to give concentrations of 50 , $25,12.5,6.25,3.125$ and $1.56 \mu \mathrm{g} / \mathrm{ml}$. $0.1 \mathrm{ml}$ suspension of bacteria and fungus in saline was added to all test tubes and were then incubated at $37 \pm 1^{\circ} \mathrm{C}$ for 24 hours (bacteria) and $25^{\circ} \mathrm{C}$ for $72 \mathrm{hrs}$ (fungi). The test mixture contained $10^{6}$ organisms $/ \mathrm{ml}(\mathrm{CFU} / \mathrm{ml})$. Macroscopic examination of inoculated culture tubes was done for turbidity. MIC was the lowest concentration at which microbial growth attenuated and no turbidity was seen in the tube. Same procedure was followed for reference drugs and the experiment was done in triplicate.

\section{Molecular Docking studies}

To get more insight into the binding interaction of the synthesized compounds, molecular docking studies were performed using Autodock Vina software. Marvin Sketch application of ChemAxon was used for drawing all the ligands employed in this study. The 3D structures of enoyl reductase-NAD ${ }^{+}$-triclosan complex (PDB id: 1C14) was procured from Protein Data Bank (www.rcsb. org) ${ }^{30}$ Before performing the docking, protein receptor was prepared by merging all the non-polar hydrogens and removing the water of crystallization using the same graphical interface. Autodock tool uses the hybrid global-local search algorithm which is a big improvement in the genetic algorithm for the best confirmations of legends. Then the protein was defined for the generation of active site, i.e. grid with specific dimensions. The parameters of grid box are given in Table 2. The
Autodock Vina uses a hybrid scoring function (empirical + knowledge based function) for evaluating binding affinity of ligands with the receptor. ${ }^{31}$

A set of 24 compounds was screened for antimicrobial activity by molecular docking simulations using PDB id : 1C14. The screening results were further compared with the in-vitro studies for their drug receptor interaction.

\section{RESULTS AND DISCUSSION}

Chemistry: In this study, 24 novel compounds incorporating benzimidazole scaffold were prepared and screened for antimicrobial activity. The reaction order for the synthesis of title compounds is given in general schemes. 2-substituted benzimidazoles were prepared by the reaction of ortho-phenylenediamine dihydrochloride with substituted carboxylic acid (Scheme 1a) and by the reaction of ortho-phenylene diamine with substituted aromatic aldehyde, carbon disulphide and cynogen bromide (Scheme 1b). Then Mannich bases were prepared by the reaction of Benzamide (active hydrogen compound), secondary amine (2-substituted benzimidazole), formaldehyde and conc. hydrochloric acid (Scheme 2). The structures of all the compounds were elucidated by spectral analysis. From IR spectra, the appearance of peaks at $3308-3310 \mathrm{~cm}^{-}$and $1400-1500 \mathrm{~cm}^{-}$ indicated the presence of $\mathrm{NH}$ of carboxamide and $\mathrm{C}=\mathrm{N}$ stretching of benzimidazole respectively. From NMR spectra, a 2 protons singlet at 4.8-4.99 ppm confirmed the presence of $\mathrm{CH}_{2}$ attached to $\mathrm{NH}$ in all the synthesized compounds. The emergence of sharp singlet at 8.0-8.9 ppm ascertained the presence of $\mathrm{NH}$ of carboxamide in all the synthesized compounds. The appearance of multiplet at $6.30-8.0 \mathrm{ppm}$ confirmed the presence of aromatic and hetero-aromatic protons. The calculated molecular weight of the synthesized compounds was comparable with observed $\mathrm{m} / \mathrm{e}$ value. Hence, the calculated values of the projected structures were found to be in good confirmity with the data obtained experimently.

Antimicrobial Evaluation of test compounds: From Table 3, it was found that amongst all synthesized compounds, $\mathbf{3 o}, \mathbf{3 q}, \mathbf{3 r}, \mathbf{3 t}, \mathbf{3} \mathbf{u}$ and $\mathbf{3} \mathbf{w}$ were most significantly active against bacterial strains as compared to standard ciprofloxacin and rest of the compounds showed moderate activity.

Structure activity relationship revealed that compound 3o with its electron withdrawing $\mathrm{Cl}$ group at 2 position of phenyl ring which is substituted at 2-position of benzimidazole showed better activity against $P$. auregenosa, $S$. aureus, B. subtilis and similar effect against $E$. coli than standard ciprofloxacin. Compound $\mathbf{3 q}$ with its elec- 
tron withdrawing $\mathrm{Cl}$ group at 4 position of benzene ring, showed improved activity against P.auregenosa, S. aureus and similar effect against E. coli and B. subtilis as compared to reference drug ciprofloxacin. Compound $3 \mathbf{r}$ with its elecron withdrawing bromo group at 2 position of phenyl ring showed better results against $P$. aerugenosa and similar effect against all the rest of the three strains when compared with ciprofloxacin. Compound $\mathbf{3 t}, \mathbf{3} \mathbf{u}$,

\begin{tabular}{|} 
Table 2: $\mathbf{x}, \mathbf{y}, \mathbf{z}$ coordinates of grid box \\
(PDB ID: 1C14) \\
\hline center_x & -1.723 \\
\hline center_y & 32.185 \\
\hline center_z & 145.55 \\
\hline size_x & 40 \\
\hline size_y & 40 \\
\hline size_z & 40 \\
\hline
\end{tabular}

3w with electron withdrawing $\mathrm{Br}, \mathrm{NO}_{2}$ and $\mathrm{F}$ groups at 4 position of phenyl ring respectively showed better activity against $P$. auregenosa and $B$. subtilis than standard drug ciprofloxacin.

From Table 4, it was found that amongst all the synthesized compounds, $\mathbf{3 o}, \mathbf{3 q}$ and $\mathbf{3 r}$ with electron withdrawing $\mathrm{Cl}$ and $\mathrm{Br}$ groups at ortho and para positions of benzene ring respectively were most significantly active against fungal strains and rest of the compounds showed moderate activity as compared to standard clotrimazole. Hence, it is concluded that substitutions on heteroaromatic rings with electron withdrawing groups like $\mathrm{Cl}, \mathrm{Br}, \mathrm{NO}_{2}$, and $\mathrm{F}$ at 2 and 4 positions of phenyl rings showed enhanced antimicrobial activity as compared to reference drugs. No inhibitory effect was found for DMSO used as control.

Molecular Docking Studies: After in vitro antimicrobial evaluation, to understand the interaction of synthesized

\begin{tabular}{|c|c|c|c|c|}
\hline Compound & $\begin{array}{c}\text { E.coli } \\
\text { (MTCC 40) }\end{array}$ & $\begin{array}{l}\text { P.aeruginosa } \\
\text { (MTCC2453) }\end{array}$ & $\begin{array}{l}\text { S.aureus } \\
\text { (MTCC 96) }\end{array}$ & $\begin{array}{l}\text { B.subtilis } \\
\text { (MTCC121) }\end{array}$ \\
\hline $3 a$ & 12.5 & 12.5 & 12.5 & 12.5 \\
\hline $3 b$ & 12.5 & 12.5 & 12.5 & 12.5 \\
\hline $3 c$ & 6.25 & 12.5 & 12.5 & 12.5 \\
\hline $3 d$ & 12.5 & 12.5 & 12.5 & 12.5 \\
\hline $3 e$ & 12.5 & 12.5 & 12.5 & 12.5 \\
\hline $3 f$ & 12.5 & 6.25 & 12.5 & 12.5 \\
\hline $3 g$ & 12.5 & 12.5 & 12.5 & 12.5 \\
\hline $3 \mathrm{~h}$ & 6.25 & 12.5 & 12.5 & 12.5 \\
\hline $3 i$ & 12.5 & 6.25 & 12.5 & 6.25 \\
\hline $3 j$ & 6.25 & 12.5 & 12.5 & 12.5 \\
\hline $3 k$ & 6.25 & 6.25 & 12.5 & 12.5 \\
\hline 31 & 6.25 & 6.25 & 12.5 & 12.5 \\
\hline $3 m$ & 12.5 & 12.5 & 6.25 & 12.5 \\
\hline $3 n$ & 6.25 & 12.5 & 6.25 & 12.5 \\
\hline 30 & 3.125 & 6.25 & 3.125 & 3.125 \\
\hline $3 p$ & 6.25 & 12.5 & 6.25 & 12.5 \\
\hline $3 q$ & 3.125 & 6.25 & 3.125 & 6.25 \\
\hline $3 r$ & 3.125 & 6.25 & 6.25 & 6.25 \\
\hline $3 s$ & 12.5 & 6.25 & 12.5 & 12.5 \\
\hline $3 t$ & 6.25 & 6.25 & 6.25 & 6.25 \\
\hline $3 u$ & 6.25 & 6.25 & 3.125 & 6.25 \\
\hline $3 v$ & 12.5 & 12.5 & 12.5 & 12.5 \\
\hline $3 w$ & 6.25 & 6.25 & 12.5 & 3.125 \\
\hline $3 x$ & 6.25 & 12.5 & 12.5 & 6.25 \\
\hline Ciprofloxacin & 3.125 & 12.5 & 6.25 & 6.25 \\
\hline Control & - & - & - & - \\
\hline
\end{tabular}




\begin{tabular}{|c|c|c|}
\hline Compound & $\begin{array}{l}\text { C. albicans } \\
\text { (MTCC 183) }\end{array}$ & $\begin{array}{c}\text { A.niger } \\
\text { (MTCC 404) }\end{array}$ \\
\hline $3 a$ & 25 & 25 \\
\hline $3 b$ & 25 & 25 \\
\hline $3 c$ & 12.5 & 12.5 \\
\hline $3 d$ & 25 & 25 \\
\hline $3 e$ & 12.5 & 25 \\
\hline $3 f$ & 25 & 25 \\
\hline $3 g$ & 25 & 25 \\
\hline $3 h$ & 25 & 25 \\
\hline $3 i$ & 12.5 & 12.5 \\
\hline $3 j$ & 25 & 25 \\
\hline $3 k$ & 12.5 & 6.25 \\
\hline 31 & 12.5 & 6.25 \\
\hline $3 m$ & 12.5 & 12.5 \\
\hline $3 n$ & 6.25 & 12.5 \\
\hline 30 & 3.125 & 3.125 \\
\hline $3 p$ & 6.25 & 12.5 \\
\hline $3 q$ & 3.125 & 6.25 \\
\hline $3 r$ & 6.25 & 3.125 \\
\hline $3 s$ & 12.5 & 25 \\
\hline $3 t$ & 6.25 & 6.25 \\
\hline $3 u$ & 6.25 & 6.25 \\
\hline $3 v$ & 25 & 25 \\
\hline $3 w$ & 6.25 & 6.25 \\
\hline $3 x$ & 6.25 & 12.5 \\
\hline Clotrimazole & 6.25 & 6.25 \\
\hline Control & - & - \\
\hline
\end{tabular}

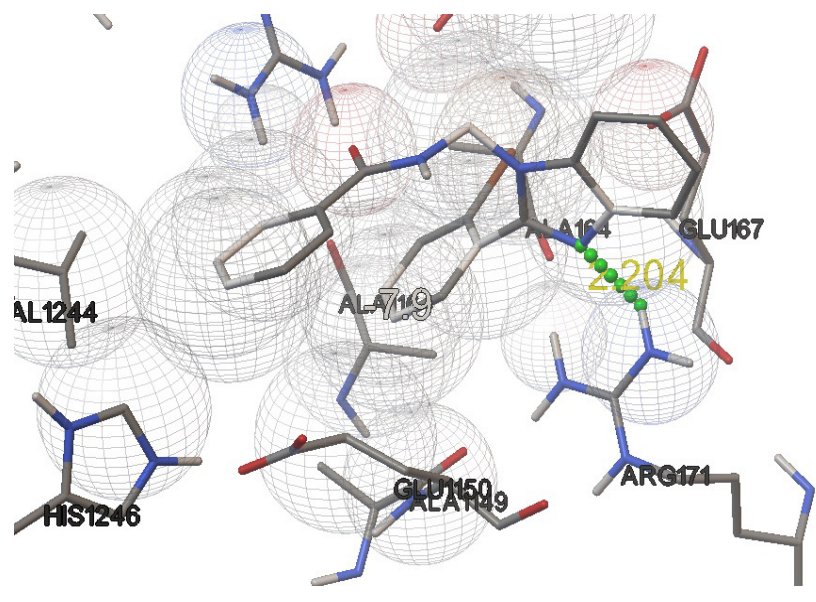

Figure 2: Binding pose for compound 3r (dock score -7.9 $\mathrm{kcal} / \mathrm{mol}$ ) within the domain of microbial receptor showing hydrogen bonding in dashed green line.

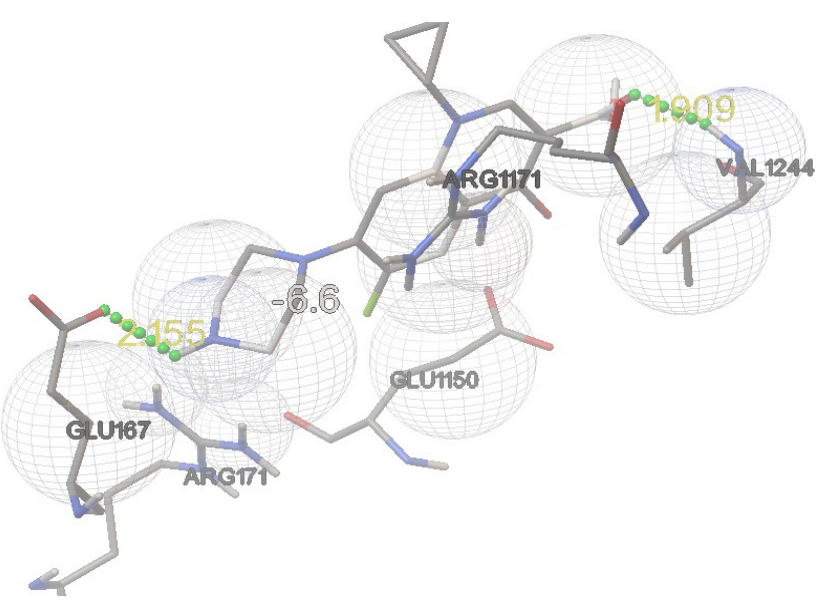

Figure 3: Binding pose for Standard Ciprofloxacin (dock score $-6.6 \mathrm{kcal} / \mathrm{mol}$ ) within the domain of microbial receptor showing two hydrogen bonding in dashed green line.

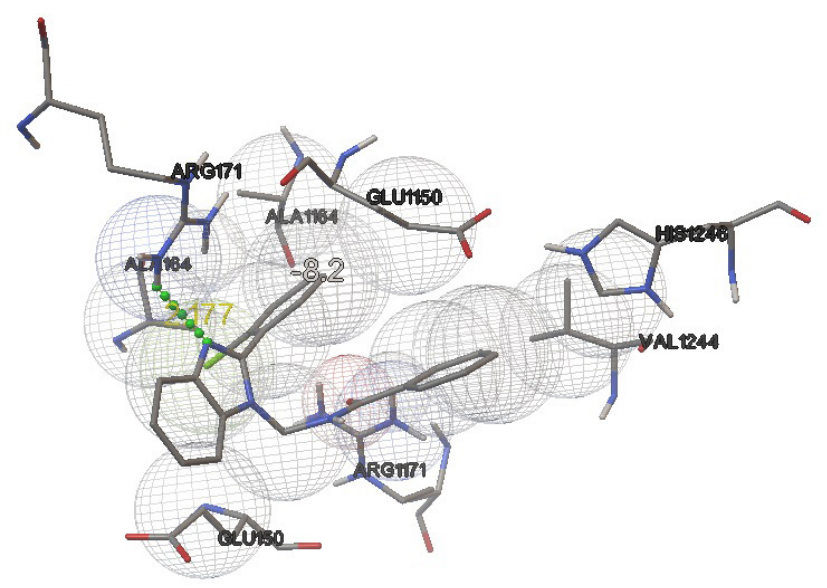

Figure 1: Binding pose for compound 30 (dock score $\mathbf{- 8 . 2}$ $\mathrm{kcal} / \mathrm{mol}$ ) within the domain of microbial receptor showing hydrogen bonding in dashed green line.

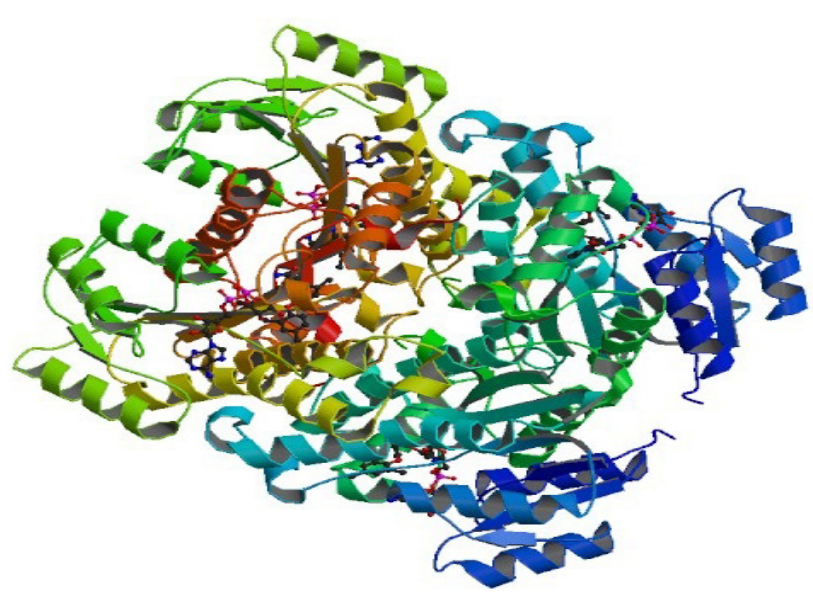

Figure 4: Secondary structure of PDB ID-1C14. 
Table 5: Data of dock score of synthesized compounds and interactive amino acids for antimicrobial

activity

\begin{tabular}{|c|c|c|c|c|}
\hline Comp & $\mathbf{R}$ & Dock score & $\begin{array}{c}\text { Number of hydrogen } \\
\text { bonds }\end{array}$ & $\begin{array}{l}\text { Amino acids involved in } \\
\text { bonding }\end{array}$ \\
\hline $3 a$ & $\mathrm{H}-$ & -7.0 & - & - \\
\hline $3 b$ & $\mathrm{CH}_{3}^{-}$ & -7.2 & - & - \\
\hline $3 c$ & $\mathrm{ClCH}_{2}^{-}$ & -7.0 & 1 & Gln1040 \\
\hline $3 d$ & $\mathrm{C}_{2} \mathrm{H}_{5}^{-}$ & -6.5 & - & - \\
\hline $3 e$ & $\mathrm{C}_{3} \mathrm{H}_{8}-$ & -7.5 & & \\
\hline $3 f$ & $\mathrm{C}_{4} \mathrm{H}_{10^{-}}$ & -7.6 & - & - \\
\hline $3 \mathrm{~g}$ & $\mathrm{NH}_{2}^{-}$ & -7.0 & 1 & Gln1040 \\
\hline $3 \mathrm{~h}$ & $\mathrm{CH}_{3}$ & -6.8 & - & - \\
\hline $3 i$ & $\mathrm{SH}-$ & -6.5 & - & - \\
\hline $3 \mathrm{j}$ & $\mathrm{SHCH}_{2}-$ & -6.5 & - & - \\
\hline $3 \mathrm{k}$ & $\mathrm{C}_{6} \mathrm{H}_{5}-$ & -7.7 & 1 & Arg171 \\
\hline $3 \mid$ & $\left(2-\mathrm{N}-\mathrm{C}_{5} \mathrm{H}_{4}\right)-$ & -7.1 & 1 & $\mathrm{Gln} 40$ \\
\hline $3 \mathrm{~m}$ & $\left(3-\mathrm{N}-\mathrm{C}_{5} \mathrm{H}_{4}\right)-$ & -7.3 & 1 & Arg1171 \\
\hline $3 n$ & $\left(2-\mathrm{OH}-\mathrm{C}_{6} \mathrm{H}_{4}\right)-$ & -8.1 & 1 & Arg171 \\
\hline 30 & $\left(2-\mathrm{Cl}-\mathrm{C}_{6} \mathrm{H}_{4}\right)-$ & -8.2 & 1 & Arg171 \\
\hline $3 p$ & $\left(3-\mathrm{Cl}-\mathrm{C}_{6} \mathrm{H}_{4}\right)-$ & -7.9 & - & - \\
\hline $3 q$ & $\left(4-\mathrm{Cl}-\mathrm{C}_{6} \mathrm{H}_{4}\right)-$ & -8.1 & - & - \\
\hline $3 r$ & $\left(2-\mathrm{Br}_{-} \mathrm{C}_{6} \mathrm{H}_{4}\right)-$ & -7.9 & 1 & Arg171 \\
\hline $3 \mathrm{~s}$ & $\left(3-\mathrm{Br}-\mathrm{C}_{6} \mathrm{H}_{4}\right)-$ & -7.8 & 1 & Gln1040 \\
\hline $3 \mathrm{t}$ & $\left(4-\mathrm{Br}-\mathrm{C}_{6} \mathrm{H}_{4}\right)-$ & -8.1 & - & - \\
\hline $3 u$ & $\left(4-\mathrm{NO}_{2}-\mathrm{C}_{6} \mathrm{H}_{4}\right)-$ & -8.0 & - & - \\
\hline $3 v$ & $\left(-\mathrm{C}_{6} \mathrm{H}_{5}-\mathrm{CH}_{2}\right)-$ & -7.9 & - & - \\
\hline $3 w$ & $\left(4-\mathrm{F}-\mathrm{C}_{6} \mathrm{H}_{4}\right)-$ & -7.8 & 1 & Arg171 \\
\hline $3 x$ & $\left(2-\mathrm{NH}_{2}-\mathrm{C}_{6} \mathrm{H}_{4}\right)-$ & -8.1 & 1 & Arg171 \\
\hline Internal ligand & ciprofloxacin & -6.6 & 2 & Glu167, Val1244 \\
\hline
\end{tabular}

compounds with microbial protein, in silico study was performed using molecular docking. Considering 1C14 as target, the series of compounds were docked to get the best in silico confirmations in the domain of $1 \mathrm{C} 14$ protein. Binding affinities of the synthesized compounds were evaluated by using docking program autodock vina, which also optimised the antimicrobial activities of synthesized compounds as possible microbial inhibitors. During analysis, H-bonding and dock score were taken as two important parameters for obtaining the hits among the set of compounds. From the in silico study, it was revealed that the compounds were having higher dock score than that of standard ciprofloxacin (-6.6 $\mathrm{kcal} / \mathrm{mol})$. The main amino acids which played a vital role in interaction are Gln1040, Arg171, Gln40, Arg 1171. Among the series, compound 30 has displayed best dock score of $-8.2 \mathrm{kcal} / \mathrm{mol}$ with $1 \mathrm{H}$-bond, length of $2.177 \mathrm{~A}^{\circ}$, shown in Figure 1. Compound 3r also displayed good dock score of $-7.9 \mathrm{kcal} / \mathrm{mol}$ with $1 \mathrm{H}$ bond, length of $2.204 \mathrm{~A}^{\circ}$, shown in Figure 2. The data of dock score and interactive amino acids of all the compounds of the series is provided in Table 5. The interactions of standard with the target protein and secondary structure of the receptor are displayed in Figure 3 and Figure 4 respectively. From the docking study, it was confirmed that the compounds substituted with halogen has displayed best interactions with the receptor among the series.

\section{CONCLUSION}

Synthesis of N-(Benzimidazol-1-yl methyl)-benzamide derivatives was done using classical mannich reaction. Their structures were elucidated by spectral 
analysis. Synthesized compounds were then screened for in vitro antibacterial and antifungal activity. Also molecular docking studies were done and their inhibitory activity was tested against PDB ID: 1C14 microbial protein. Both the theory (Docking) and practice (MIC values) demonstrate that N-(Benzimidazol-1-yl methyl)-benzamide derivatives act as potent inhibitor of 1C14 microbial protein. All the synthesized derivatives showed momentous antimicrobial activity against bacterial strains E.coli (MIC 12.5-3.125 $\mu \mathrm{g} /$ $\mathrm{ml}$ ), P. aeruginosa (MIC 12.5-3.125 $\mu \mathrm{g} / \mathrm{ml}$ ), S.aureus (MIC 12.5-3.125 $\mu \mathrm{g} / \mathrm{ml}$ ) and B.subtilis (MIC 12.5$3.125 \mu \mathrm{g} / \mathrm{ml}$ ) in comparison to reference drug ciprofloxacin and against fungal strains, C.albicans (MIC 25-3.125 $\mu \mathrm{g} / \mathrm{ml}$ ) and A. niger (MIC 25-3.125 $\mu \mathrm{g} /$ $\mathrm{ml}$ ) when compared with clotrimazole as standard antimicrobial agent. Amongst all the synthesized compounds, $3 \mathbf{s}, \mathbf{3 q}, \mathbf{3 r}, \mathbf{3 t}, \mathbf{3 u}$ and $\mathbf{3 w}$ with its electron withdrawing substitutions (2-chloro, 4-chloro, 2-bromo, 4-bromo, 4-nitro and 4-flouro groups respectively) on aromatic rings were the most active compounds against the bacterial strains. Compounds 3o and 3q with chloro substitution at 2 and 4 position of phenyl ring respectively and compound $3 \mathbf{r}$ with its bromo substitution at 2 position of phenyl ring were the most active compounds against fungal strains. Hence it is concluded that N-(Benzimidazol1-yl methyl)-benzamide derivatives with electron withdrawing substituents could be used for designing more potent antimicrobial agents. Further studies of these derivatives are highly suggested to judge the safety of novel compounds.

\section{ACKNOWLEDGEMENT}

The authors are thankful to Management, Chitkara University, Punjab for providing necessary facilities to carry out this work.

\section{CONFLICT OF INTEREST}

The author declares that there is no conflict of interests.

\section{ABBREVIATIONS USED}

MS: Mass Spectrometry; FTIR: Fourier transform infrared spectroscopy; NMR: Nuclear magnetic resonance; IP: Indian Pharmacopoeia; TLC: Thin Layer Chromatography; DMSO-d 6 : Deuterated Dimethyl Sulphoxide; MIC: Minimum inhibitory concentration; Int.lgn: Internal ligand; PDB: Protein Data Bank; MTCC: Microbial Type culture collection.

\section{REFERENCES}

1. Goker H, Kus C, Boykin DW, Yildiz SN and Altanlar N. Synthesis and Antimicrobial Activity of Some New 2-Phenyl-N-substituted Carboxamido1H-benzimidazole Derivatives. Arch Pharm Med Chem. 2001;334(5):14852.

2. Woodford $\mathrm{N}$ and Ellington MJ. The emergence of antibiotic resistance by mutation. Clinical Microbiology and Infection : the Official Publication of the Eur Society Clinical Microbiology and Infectious Diseases. 2007;13(1):5-18.

3. Pawar NS, Dalal DS, Shimpi SR, Mahulikan PP. Studies of antimicrobial activity of $\mathrm{N}$-alkyl and $\mathrm{N}$-acyl 2-(4-thiazolyl)-1H-benzimidazoles. Eur J Pharm Sci. 2004;21(2-3):115-8

4. Goker H, Kus C, Boykin DW, Yildiz S and Altanlar N. Synthesis of some new 2-substituted-phenyl-1H-benzimidazole-5-carbonitriles and their potent activity against Candida species. Bioorg Med Chem. 2002;10(8):2589-96.

5. Mohammad BG, Hussein MA, Abdel-Alim AA and Hashem M. Synthesis and antimicrobial activity of some new 1-alkyl-2-alkylthio-1,2,4triazolobenzimidazole derivatives. Arch Pharm Res. 2006;29(1):26-33.

6. Deep A, Jain S, Sharma PC, Mittal SK, Phogat P and Malhotra M. Synthesis, characterization and antimicrobial evaluation of 2, 5-disubstituted-4thiazolidinone derivatives. Arabian J of Chem. 2014;7(3):287-91.

7. Nakano $H$, Inoue $T$, Kawasaki N, Miyatka $H$, Miyatka $H$, Taguchi $T$ and Satoh T. Synthesis and Biological activites of novel antiallergic agents with 5-lipooxygenase inhibiting action. Bioorganic Med Chem. 2000;8(2):37380.

8. White AW, Almassy R, Calvert AH and Golding BT. Resistance modifying agents, Synthesis and Biological properties of Benzimidazole inhibitors of DNA repair enzyme Poly(ADP Ribose) polymerase. J Med Chem. 2000;43:4084-97.

9. Zhu Z, Lippa B and Drach JC. Design Synthesis and biological evaluation of tricyclic nucleosides (Dimensional probes) as analogues of certain antiviral polyhalogenated Benzimidazole Ribonucleosides. J Med Chem. 2000;43(12):2430-7

10. Bariwal JB, Shah AK, Kathiravan MK, Somani RS, Jagtap JR and Jai KS. Synthesis and antiulcer activity of novel pyrimidylthiomethyl and Pyrimidylsulfinylmethyl benzimidazoles as potential reversible proton pump inhibitors. Ind J Pharm Ed Res. 2008;42(3):225-31.

11. Mariappan G, Hazarika R, Alam F, Karki R, Patangia U and Nath S. Synthesis and biological evaluation of 2-substituted benzimidazole derivatives. Arabian $\mathrm{J}$ of Chem. 2015;8:715-9.

12. Spasov AA, Yozhitsa IN and Bugaeva LI. Benzimidazole derivatives: Spectrum of Pharmacological activity and toxicological properties (a review). Pharm Chem Journal. 1999;33(5):232-43.

13. March J. Advanced Organic Chemistry: Reaction Mechanisms and Structure, Fourth edition. A. Wiley-Interscience Publication, New York; John Wiley and Sons:900-902.1992.

14. Achar KC, Hosamani KM and Seetharamareddy HR. In vivo analgesic and anti-inflammatory activities of newly synthesized benzimidazole derivatives. Eur J Med Chem. 2010;45(5):2048-54.

15. Malinka W, Swiatek P, Filipek B, Sapa J, Jezierska A and Koll A. Synthesis, analgesic activity and computational study of new isothiazolopyridines of mannich base type. Farmaco. 2005;60(11-12):961-8.

16. Kalluraya B, Chimbalkar RM and Hedge JC. Anticonvulsant activity of nicotinyl/isonicotinyl substituted 1,2,4-triazol-5-thione Mannich bases. Indian Journal of Heterocyclic Chemistry. 2005;15(1):15-8.

17. Koksal M, Gokhan N, Kupeli E, Yesilada E and Erdogan H. Analgesic and anti-inflammatory activities of some new mannich bases of 5- nitro-2benzoxazolinones. Archives of Pharmacal Research. 2007;30(4):419-24.

18. IvanovaY, Momekov G, Petrov O, Karaivanova M and Kalcheva V. Cytotoxic Mannich bases of 6-(3-aryl-2-propenoyl)-2-(3H)-benzoxazolones. Eur J Med Chem. 2007;52(11-12):1382-7.

19. Gul HI, Vepsalainen J, Gul M, Erciyas E and Hanninen O. Cytotoxic activities of mono and bis mannich bases derived from acetophenone against Renca and Jurkat cells. Pharmaceutica Acta Helvetiae. 2000;74(4):393-8.

20. Vashishtha SC, Zello GA, Nienaber KH. Cytotoxic and anticonvulsant aryloxy aryl mannich bases and related compounds. Eur J Med Chem. $2004 ; 39(1): 27-35$. 
21. Ashok M, Holla BS and Poojary BC. Convenient one pot synthesis and antimicrobial evaluation of some new Mannich bases carrying 4-methylthiobenzyl moiety. Eur J Med Chem. 2007;42(8):1095-1.

22. Pandeya SN, Sriram D, Nath G and De Clercq. Synthesis, antibacterial, antifungal and anti-HIV activities of norfloxacin Mannich bases. Eur J Med Chem. 2000;35(2):249-55.

23. Singh BN, Shukla SK and Singh M. Synthesis and biological activity of sulphadiazine schiff's bases of isatin and their N-Mannich bases. Asian J of Chemistry. 2007;19(7):5013-8.

24. Babbar R, Pathak DP, Jain N, Jain S. Synthesis and anti-inflammatory activity of mannich bases of nicotinamide with diclofenac and mefenamic acid. Der Pharma Chemica. 2012;4(5):2024-8.

25. Furniss BS, Hannaford AJ, Smith PWG and Tatchell R. Vogel's Text book of Practical Organic Chemistry. $5^{\text {th }}$ edition. Longman scientific and technical England;1989.

26. Devmurari VP, Pandey S, Goyani MB, Jivani NP. Synthesis and antibacterial evaluation of 2- some substituted benzimidazole-1-carbodithionate derivatives. Inter J Chem Tech Res. 2010;2(1):598-605.
27. Simonov AM, Anisimova VA. Synthesis and transformation of 2- amino benzimidazoles. (Review). Chemistry of Heterocyclic Compounds. 1979;15(7):705-23.

28. Wang ML, Liu BL. Synthesis of 2- mercaptobenzimidzole from the reaction of o-phenylene diamine and carbon disulphide in the presence of potassium hydroxide. Journal of Chinese Institute of Chemical Engineers. 2007;38(2):161-7.

29. Pharmacopoeia. Pharmacopoeia of India. vol. II. Ministry of Health Department, Govt. of India, New Delhi :1996.

30. Shah UA, Wagh NK, Deokar HS, Kadam SS, and Kulkarni VM. Design, synthesis, pharmacological screening and molecular docking of biphenyl analogues as antiinflammatory agents (Part-I). Journal of Pharma and Bio Sciences. 2010;1(4):501.

31. Trott O, Olson AJ. AutoDock Vina: improving the speed and accuracy of docking with a new scoring function, efficient optimization and multithreading. J Comput Chem. 2010;31(2):455-61.

\section{SUMMARY}

- A series of N-benzimidazol-1-yl-methyl-benzamide derivatives (3a-3x) were synthesized by Mannich reaction and evaluated for in vitro antimicrobial activity against various bacterial and fungal strains. The novel target compounds were further characterized by spectral and analytical techniques. Among the synthesized derivatives, 3o N-[2-(2-chloro-phenyl)-benzimidazol-1-ylmethyl]-benzamide, 3q N-[2-(4-chloro-phenyl)-benzimidazol-1ylmethyl]-benzamide and 3r N-[2-(2-bromo-phenyl)-benzimidazol-1-ylmethyl]-benzamide were found to be most effective antimicrobial compounds. Clotrimazole and ciprofloxacin were used as reference antimicrobial agents. Molecular docking studies were also performed to describe the interaction of the title compounds with microbial protein. Hence it is summarized that $\mathrm{N}$-(Benzimidazol-1-yl methyl)-benzamide derivatives with electron withdrawing substituents might be used for designing more effective antimicrobial agents. 